\title{
Final results on atmospheric muon neutrino oscillations with MACRO
}

\author{
Miriam Giorgini*, for the MACRO Collaboration ${ }^{\dagger}$ \\ University of Bologna and INFN, V.le Berti Pichat 6/2, I-40127 Bologna, Italy \\ E-mail: miriam.giorgini@bo.infn. itit
}

ABSTRACT: The final analysis of atmospheric neutrino events collected with the MACRO detector is presented. Three different classes of events, generated by neutrinos in different energy ranges, are studied in terms of rates, angular distributions and estimated energies. The results are consistent for all the subsamples and indicate a flux deficit depending on energy and pathlength of neutrinos. The no oscillation hypothesis is excluded at $\sim 5 \sigma$, while the hypothesis of $\nu_{\mu} \longleftrightarrow \nu_{\tau}$ oscillation gives a satisfactory description of all data. The parameters with higher probability in a two flavor scenario are $\Delta m^{2}=2.3 \cdot 10^{-3}$ $\mathrm{eV}^{2}$ and $\sin ^{2} 2 \theta=1$.

\section{Introduction}

MACRO was a large multipurpose underground detector [i] Gran Sasso Laboratory from 1989 till the end of 2000. Though it was optimised to search for the supermassive GUT magnetic monopoles [2], MACRO obtained important results on atmospheric $\nu_{\mu}$ oscillations and performed $\nu_{\mu}$ astronomy studies [3] for WIMPs [i⿱亠幺厶] $]$ and for low energy $\bar{\nu}_{e}$ from stellar gravitational collapses.

The detector started data taking with part of the apparatus in 1989; it was completed in 1995 and was running in its final configuration until December 2000.

In the following, after a detailed analysis of the present situation of the different Monte Carlo (MC) simulations of the atmospheric neutrino flux, the final neutrino oscillation results obtained by MACRO are summarised.

\section{The MACRO detector}

MACRO was a large rectangular box, $76.6 \mathrm{~m} \times 12 \mathrm{~m} \times 9.3 \mathrm{~m}$, divided longitudinally in six supermodules and vertically in a lower part (4.8 m high) and an upper part (4.5 m high),

${ }^{*}$ Speaker.

${ }^{\dagger}$ For the full list of the Collaboration see the second paper of ref. [1] [1] 


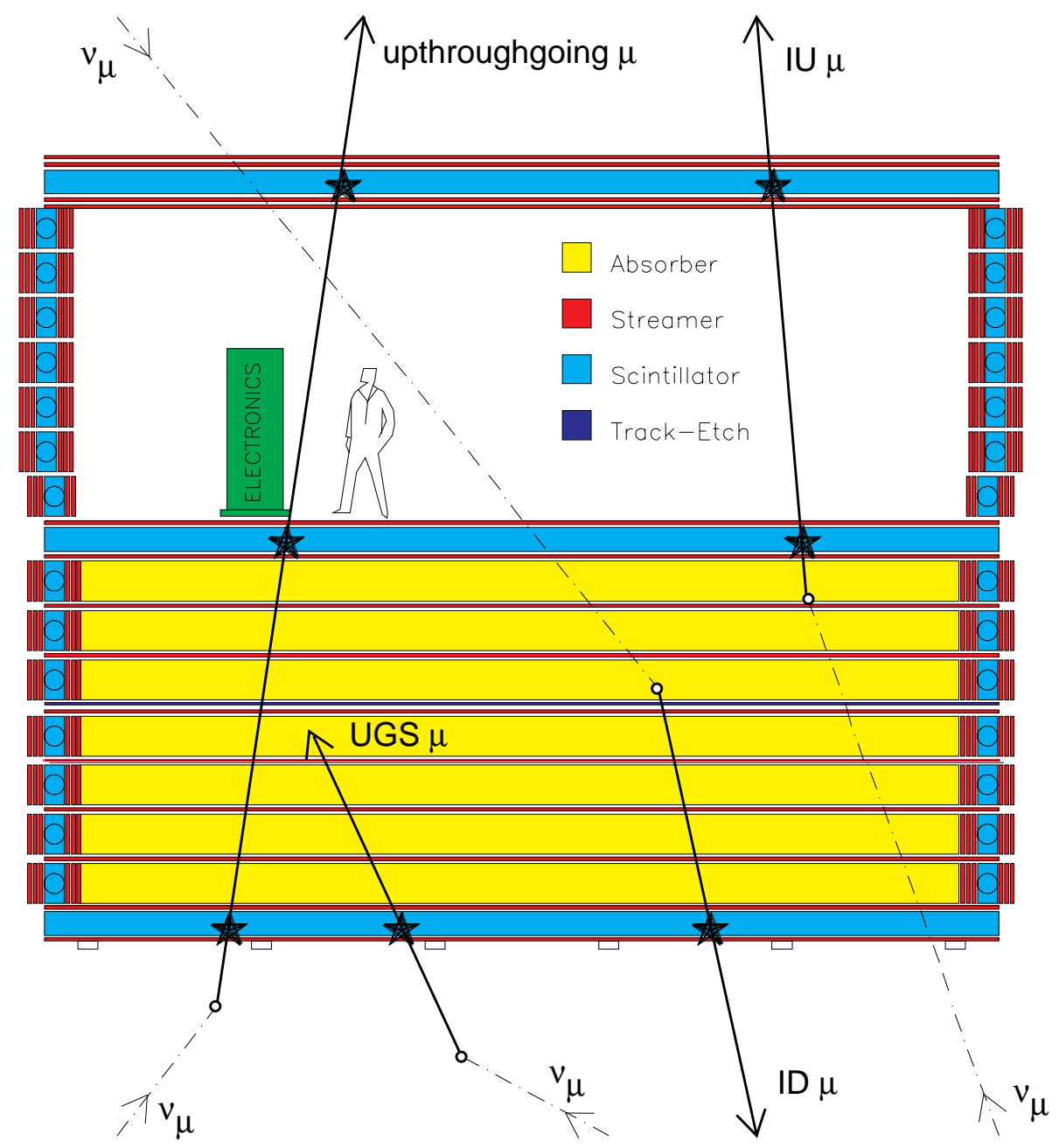

Figure 1: Vertical section of the MACRO detector, which shows the event topologies induced by $\nu_{\mu}$ interactions in or around the detector: Upthroughgoing $=$ upward throughgoing $\mu, \mathrm{IU}=$ semicontained Internal Upgoing $\mu, \mathrm{ID}=$ Internal Downgoing $\mu, \mathrm{UGS}=$ Upgoing Stopping $\mu$.

Fig. ㄱ.1‥ It had three types of detectors which gave redundancy of informations: liquid scintillation counters, limited streamer tubes and nuclear track detectors. These last detectors were used only for rare particle searches.

For muon and for neutrino physics, the streamer tubes were used for muon tracking and the liquid scintillation counters for fast timing. The lower part of the detector was filled with trays of crushed rock absorbers alternating with streamer tube planes; the upper part was open and contained the electronics [i]

There were 10 horizontal planes of streamer tubes in the bottom half of the detector, and 4 planes at the top, all with wires and $26.5^{\circ}$ stereo strips readout. Six vertical planes of streamer tubes and one layer of scintillators covered each side of the detector. The scintillator system consisted of three layers of horizontal counters, and of the mentioned vertical layer along the sides of the detector.

The combination of the informations from the streamer tubes and from the scintillators 
allowed tracking with a precision of $1 \mathrm{~cm}$ over path lengths of several meters, and timing with a precision of $600 \mathrm{ps}$. The detector provided a total acceptance $S \Omega \simeq 10000 \mathrm{~cm}^{2} \mathrm{sr}$ for an isotropic flux of particles.

Fig. 'i-i is a vertical section of the detector; it shows a general view of the detector and gives a sketch of the different topologies of detected neutrino-induced muon events used to study neutrino oscillations: Upthroughgoing muons, Internal Upgoing muons (IU), Upgoing Stopping muons (UGS) and Internal Downgoing muons (ID).

\section{Atmospheric neutrinos}

Atmospheric neutrinos are the result of a decay chain starting with the interactions of high energy primary cosmic rays in the upper atmosphere. Each interaction produces a large number of pions and kaons, which decay yielding muons and muon neutrinos; also the muons decay yielding $\nu_{\mu}$ and $\nu_{e}$. The ratios of their numbers are $N_{\nu_{\mu}} / N_{\nu_{e}} \simeq 2$ and $N_{\nu} / N_{\bar{\nu}} \simeq 1$. The atmospheric neutrinos are produced at about $10-20 \mathrm{~km}$ above ground. They have energies ranging from a fraction of $\mathrm{GeV}$ up to more than $100 \mathrm{GeV}$ and travel distances $L$ from few tens of $\mathrm{km}$ up to $13000 \mathrm{~km}$. An underground detector is "crossed" by a neutrino flux from all directions and it can make oscillation studies for $1<L / E_{\nu}<10^{4}$ $\mathrm{km} / \mathrm{GeV}$.

MACRO detected upgoing muon neutrinos via charged current interactions, $\nu_{\mu} \rightarrow \mu$.

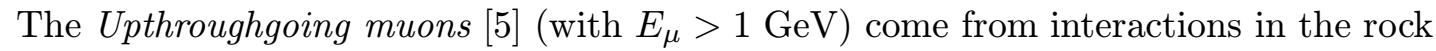
below the detector of muon neutrinos with an average energy $\left\langle E_{\nu}\right\rangle \sim 50 \mathrm{GeV}$. The tracking is performed with streamer tubes hits; the time information, provided by scintillation counters, allows the determination of the direction (versus) by the Time of Flight (ToF) method.

The Internal Upgoing muons (IU) [鿒] come from $\nu_{\mu}$ interactions inside the lower apparatus. Since two scintillation counters are intercepted, the ToF method is applied to identify the upward going muons. The average parent neutrino energy for these events is $\sim 2.4 \mathrm{GeV}$.

The UpGoing Stopping muons (UGS) [6. the detector yielding upgoing muon tracks stopping in the detector; the Internal Downgoing muons (ID) [í;] are due to $\nu_{\mu}$ induced downgoing tracks with vertex in the lower MACRO. The events are found by means of topological criteria; the lack of time information prevents to distinguish between the two subsamples. The average parent neutrino energy for these events is $\sim 2.2 \mathrm{GeV}$.

\subsection{Monte Carlo simulations}

The measured data for each topology were compared with different MC simulations. In the past we used the neutrino flux computed by the Bartol group [i] and the parton distribution given in ref. [8]. For the low energy channels the cross sections in [9i] were used; the propagation of muons to the detector was done using the energy loss calculation of ref. [1] $1 \overline{0}]$. The total systematic uncertainty in the predicted flux of upthroughgoing muons, adding in quadrature the errors, was estimated to be $\pm 17 \%$; this is mainly a scale 


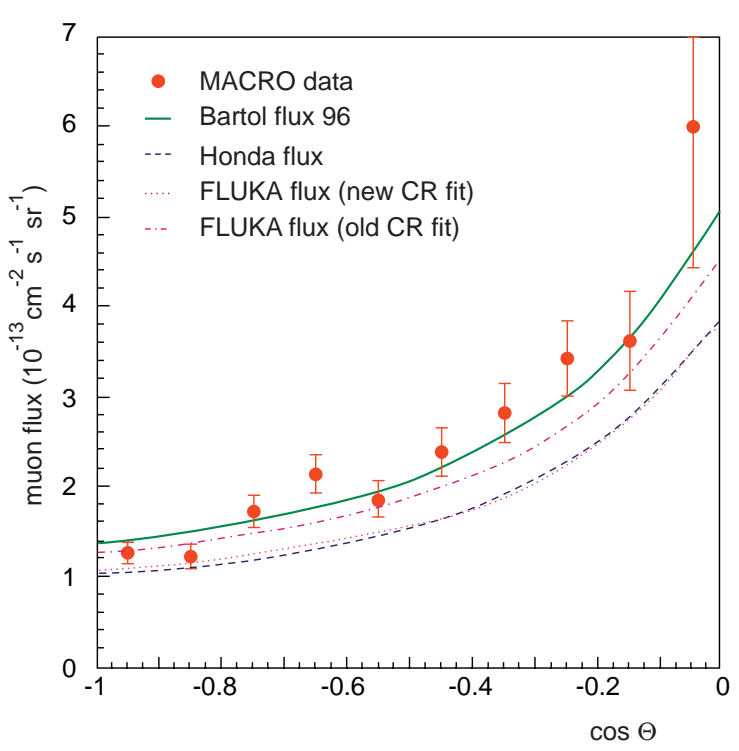

(a)

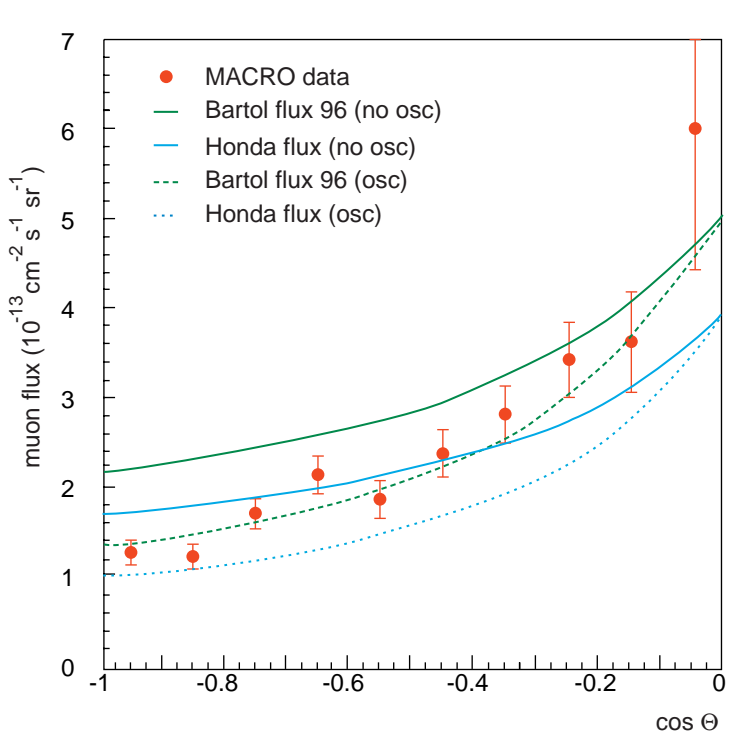

(b)

Figure 2: (a) Zenith distribution of upthroughgoing muons compared with the predicted fluxes obtained from MC simulations in ref. [7] maximal mixing. The solid line is the Bartol flux, the dotted and the dashed lines are the new FLUKA and HKKM fluxes; the dotted-dashed line is the FLUKA flux with the old fit to the cosmic ray data. (b) Comparison of our measurements with the Bartol and the HKKM oscillated and non oscillated fluxes.

error which does not change the shape of the angular distribution. The error on the shape of the distribution is $\sim 5 \%$. The detector was simulated using the GEANT package [i $\left.{ }_{1}^{1} \overline{1}\right]$.

Recently new improved MC predictions were made available by the HKKM [i] FLUKA [1] decays and of neutrino interactions, improved hadronic model and new fits of the primary

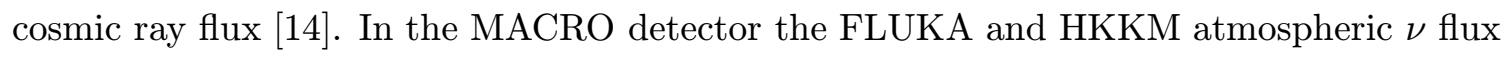
produce an event rate lower by about $25 \div 30 \%$, while the shapes of the angular distributions differ by $\sim 5 \%$. The new calculations HKKM and FLUKA show good agreement when they use the same fit to the primary CR flux [1] model.

In Fig. 12. our data are compared with the oscillated Bartol, HKKM and FLUKA calculations, the last using both the new and the old CR fits. All predicted curves are for maximal mixing and $\Delta m^{2}=2.3 \cdot 10^{-3} \mathrm{eV}^{2}$.

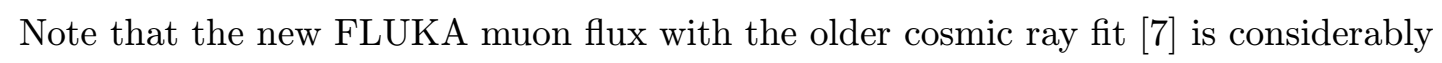
above the new FLUKA flux with the new CR fit.

If neutrino oscillations with the preferred parameters are taken into account, the predicted normalization is about $38 \%$ lower than the MACRO data. Thus the recent fit to the primary CR energy distribution yields a neutrino flux too low above $100 \mathrm{GeV}$ to account for our measurement of upthroughgoing muons.

Thus the Bartol MC may very probably still be used for the prediction of the absolute 
flux, besides of the shape of the angular distribution. In the following we shall use the predictions of this MC. In Fig. oscillated predictions of Bartol and HKKM flux.

\section{MACRO results on atmospheric neutrinos.}

The events measured for the three topologies and the $L / E_{\nu}$ distribution for upthroughgoing muons deviate from Monte Carlo expectations without oscillations. For each detected topology, Table $\prod_{1}^{1}$ gives the numbers of the measured events and of the predicted events by the oscillation and no oscillation hypotheses.

\begin{tabular}{lccc}
\hline & Upthrough. & IU & ID+UGS \\
\hline Measured events & $857 \pm 29_{\text {stat }}$ & $157 \pm 12_{\text {stat }}$ & $262 \pm 16_{\text {stat }}$ \\
Expected events (no osc) & 1169 & 235 & 315 \\
Expected events (osc) & 820 & 135 & 238 \\
\hline
\end{tabular}

Table 1: For each topology the table gives the number of events measured and predicted by the oscillation and no oscillation hypotheses

\subsection{Upthroughgoing muons}

The data were collected during the running period from March 1989 to April 1994 with the detector under construction and during the runs with the complete detector from 1994 until December 2000 (livetime 5.52 yrs). Since the total livetime normalised to the full configuration is $6.16 \mathrm{yrs}$, the statistics is largely dominated by the full detector run.

A large number of possible systematic effects and backgrounds that could affect the measurements were studied.

One of the main cuts to remove background requires that the position of a muon hit in each scintillator, as determined from the timing within the scintillator counter, agrees within $\pm 70 \mathrm{~cm}$ with the position indicated by the streamer tube track.

In order to reduce the background due to downgoing muons passing near MACRO and producing low energy upgoing particles which could appear neutrino-induced upgoing muons [1] the lower part of the detector.

A large number of nearly horizontal $(\cos \Theta>-0.1)$ upgoing muons have been observed coming from azimuth angles between $-30^{\circ}$ and $120^{\circ}$. In this direction, the rock overburden is insufficient to remove nearly horizontal downgoing muons which have scattered in the mountain and appear as upgoing. This region was excluded for real events and for MC simulations.

The direction of muons crossing MACRO is determined by the time of flight method, between two layers of scintillators. The measured muon velocity is calculated with the convention that downgoing muons have $1 / \beta=+1$ and upgoing muons have $1 / \beta=-1$. We selected upwardgoing muons requiring $-1.25 \leq 1 / \beta \leq-0.75$. 


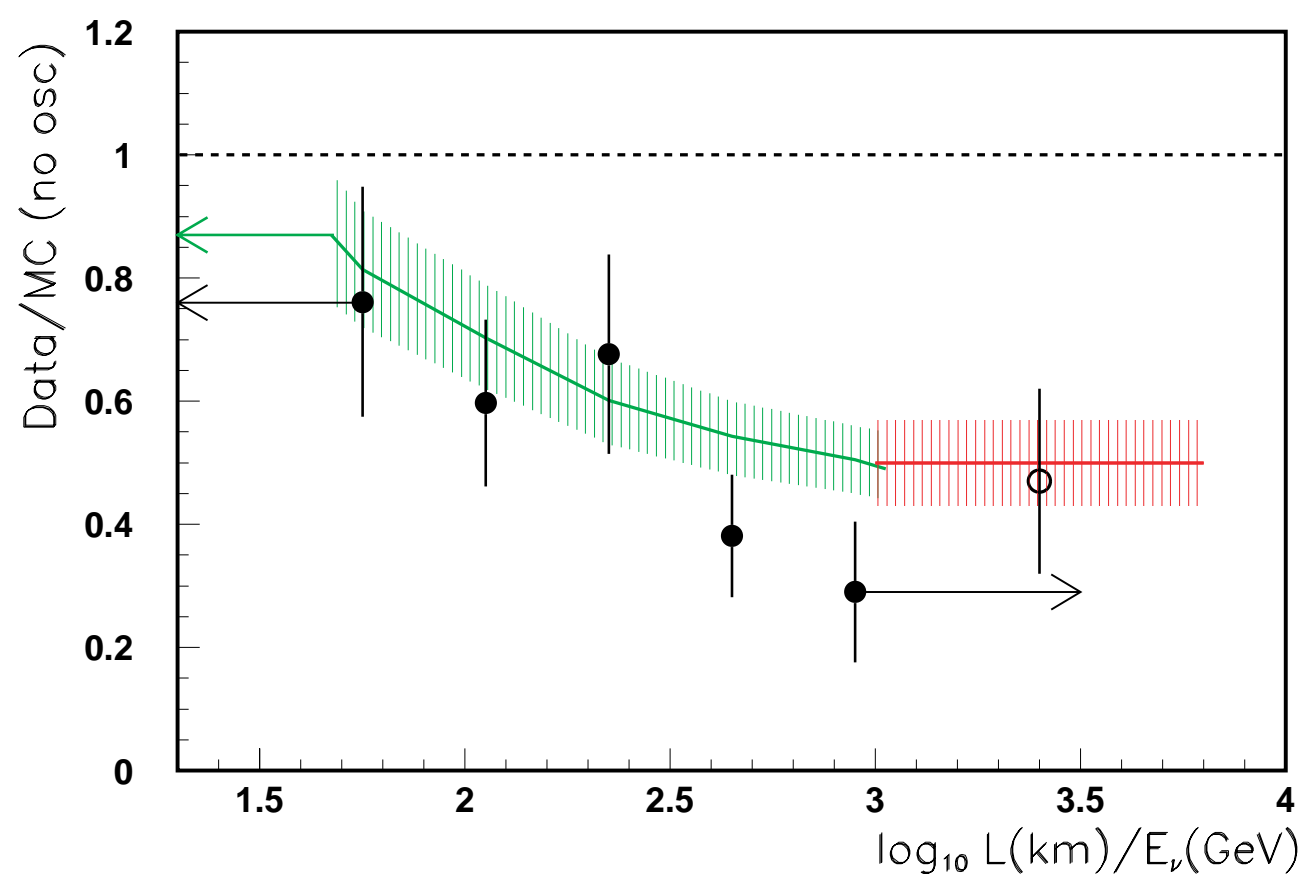

Figure 3: Ratio Data/MC as a function of estimated $L / E_{\nu}$ for the upthroughgoing muon sample (black circles) and the IU $\mu$ (open circle). The full lines represent the MC predictions assuming $\sin ^{2} 2 \theta=1$ and $\Delta m^{2}=2.3 \cdot 10^{-3} \mathrm{eV}^{2}$. The shaded regions represent the $12 \%$ uncertainties in the oscillated MC predictions.

The measured data, Table ${ }_{1}^{1}$ and Fig. $2 \bar{b}$, deviate in shape and in absolute value from the Bartol MC predictions. This was first pointed by MACRO in 1995 [i] $\left.{ }_{1}^{1}\right]$ ].

Assuming no oscillations, the number of expected upthroughgoing muon events integrated over all zenith angles from Bartol without oscillations is 1169; the measured number is 857, Table $\underline{1}_{1}$. Thus the ratio of the observed number of events to the Bartol expectation is 0.73 .

\subsection{1 $L / E_{\nu}$ distribution}

Since the neutrino oscillation probability explicitly depends on their energy $E_{\nu}$ and travelled distance $L$ between the production and the interaction points, an estimate of the energy of upthroughgoing muons provided an important contribution to the understanding of the oscillation mechanism. Although a significant fraction of the neutrino energy is absorbed by the hadrons in the interaction and then lost during the muon propagation to the detector, MC simulations show a linear correlation between the parent neutrino energy $E_{\nu}$ and the muon residual energy $E_{\mu}$ at the detector level.

Since MACRO was not equipped with a magnet, the only way to experimentally estimate the muon energy was through the Multiple Coulomb Scattering (MCS) of muons in the absorbers. This analysis was based on the relation between the r.m.s. of the lateral displacement and the momentum of relativistic particles crossing a layer of material.

A first analysis was performed using the digital information from the streamer tubes 
[1] $\left[\begin{array}{l}1 \\ \overline{1}\end{array}\right]$ with an intrinsic space resolution $\sigma_{x} \simeq 1 \mathrm{~cm}$. This analysis showed the feasibility of the method for muon energies up to $\sim 10 \mathrm{GeV}$. To improve the space resolution, the streamer

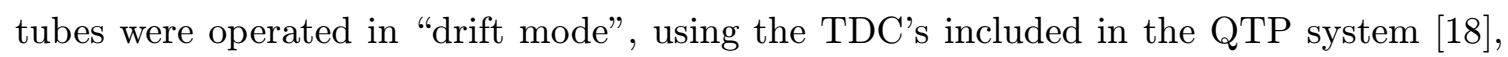
originally designed for the search for magnetic monopoles. To check the electronics and the feasibility of the analysis, two "test beams" were performed at the CERN PS-T9 and SPS$\mathrm{X} 7$ beams. The space resolution achieved was $\simeq 3 \mathrm{~mm}$, a factor of 3.5 better than in the previous analysis $[\overline{1} \overline{1} \overline{9}]$. For each muon, 7 MCS sensitive variables were given in input to a Neural Network, previously trained to estimate $E_{\mu}$ with MC events of known input energy crossing the detector at different zenith angles. $E_{\nu}$ was estimated by MC simulations from the muon energy, with a resolution of $150 \%$.

For each event, the neutrino travelled distance $L$ was measured with a $\sim 3 \%$ precision by using the reconstructed zenith angle of the tracked muon.

The distribution of the ratio $R=$ Data/MCno osc obtained by this analysis is plotted in Fig. $\underline{\underline{3}}$, as a function of $L / E_{\nu}[\underline{1} \overline{1} \overline{7} \overline{1}]$.

The black points are the data with their statistical errors. The continuous line represents the MC prediction for $\nu_{\mu} \longleftrightarrow \nu_{\tau}$ oscillations with maximal mixing and $\Delta m^{2}=$ $2.3 \cdot 10^{-3} \mathrm{eV}^{2}$, the shaded area includes a $12 \%$ point-to-point error associated to the MC prediction. In the same figure, the black square and the associated MC prediction refer to the IU events (see $\left.\overline{4} .2_{1}\right)$.

To quantify the independent sensitivity of this analysis to neutrino oscillations, a MC was used to define the best parameter to separate the oscillation hypothesis from the no oscillation one. The ratio $N_{\text {low }} / N_{\text {high }}$, where $N_{\text {low }}$ and $N_{\text {high }}$ are the numbers of events with $E_{\nu}<30 \mathrm{GeV}$ and $E_{\nu}>130 \mathrm{GeV}$ respectively, gives the best performance. The measured ratio is $R_{\text {meas }}=0.85 \pm 0.16_{\text {stat }}$; the $\mathrm{MC}$ prediction in case of no oscillations is $R_{0}=$ $1.50 \pm 0.25_{t h+s y s}$, while for $\Delta m^{2}=2.3 \cdot 10^{-3} \mathrm{eV}^{2}$ and $\sin ^{2} 2 \theta=1, R_{\tau}=1.00 \pm 0.17_{t h+s y s}$ (see Table $\left.{ }^{1} \bar{i}\right)$.

\subsection{2 $\nu_{\mu} \longleftrightarrow \nu_{\tau}$ or $\nu_{\mu} \longleftrightarrow \nu_{\text {sterile }}$ ?}

The weak potential in matter could produce a phase shift that could modify the oscillation pattern if the oscillating neutrinos have different interactions with matter [20 $\left.{ }_{0}^{0}\right]$. The matter effect could discriminate between different neutrino channels: it could be important for $\nu_{\mu} \longleftrightarrow \nu_{e}$ and for $\nu_{\mu} \longleftrightarrow \nu_{\text {sterile }}$ oscillations, while for $\nu_{\mu} \longleftrightarrow \nu_{\tau}$ there is no matter effect ( $\nu_{\mu}$ and $\nu_{\tau}$ have the same weak potential in matter).

In the $\nu_{\mu} \longleftrightarrow \nu_{\text {sterile }}$ oscillation scenario, the matter effect changes the shape of the angular distribution and the total number of events with respect to the vacuum oscillations. Large matter effects are expected for neutrinos crossing the Earth, due to the long path length and to the increase of the density in the Earth core. In absence of resonances, due to particular values of the oscillation parameters, the matter effect produces a reduction of the oscillation effect, giving a prediction similar to the no oscillation scenario, particularly for directions near the vertical.

A statistically powerful test is based on the ratio between the events with $-1<\cos \Theta<$

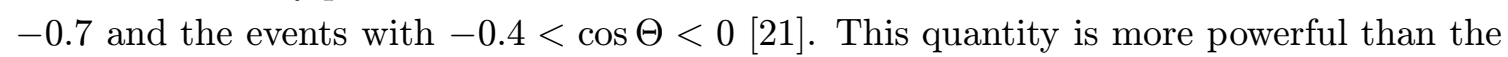
$\chi^{2}$ because the data are binned to maximise the difference between the two hypotheses 
and the ratio is sensitive to the sign of the variation. The angular regions were chosen by MonteCarlo methods in order to have the best discrimination between the $\nu_{\mu} \longleftrightarrow \nu_{\tau}$ and $\nu_{\mu} \longleftrightarrow \nu_{\text {sterile }}$ oscillations.

In this ratio, most of the theoretical uncertainties on neutrino flux and cross sections cancel. The measured ratio is $R=1.38 \pm 0.12$.

For $\Delta m^{2}=2.3 \cdot 10^{-3} \mathrm{eV}^{2}$ and maximal mixing, the minimum expected value of the ratio for $\nu_{\mu} \longleftrightarrow \nu_{\tau}$ oscillations is $R_{\tau}=1.61$; for $\nu_{\mu} \longleftrightarrow \nu_{\text {sterile }}$ oscillations one expects $R_{\text {sterile }}=2.03$. The maximum probabilities to find a value of $R_{\tau}$ and of $R_{\text {sterile }}$ smaller than $R_{\text {expected }}$ are $7.2 \%$ and $1.5 \cdot 10^{-4}$ respectively. Hence, the ratio of the maximum probabilities is $P_{\tau} / P_{\text {sterile }} \simeq 480$, so that $\nu_{\mu} \longleftrightarrow \nu_{\text {sterile }}$ oscillations (with any mixing) are excluded at $99.8 \%$ C.L. compared to the $\nu_{\mu} \longleftrightarrow \nu_{\tau}$ channel with maximal mixing and $\Delta m^{2}=2.3 \cdot 10^{-3} \mathrm{eV}^{2}$.

\subsection{Low energy events}

These events were mainly due to $\nu_{\mu} \mathrm{CC}$ interactions, with a contribution from $\mathrm{NC}$ and $\nu_{e}(\sim 13 \%$ for IU and $\sim 10 \%$ for UGS+ID). The data concern only the running period with the detector in the full configuration from April 1994 to December 2000. Due to the difference between the topologies of the low energy events, two separate analyses were performed.

The identification of Internal Upgoing (IU) events was based both on topological criteria and ToF measurements. The IU sample corresponds to an effective livetime of 5.8 yrs. The basic request was the presence of a streamer tube track reconstructed in space matching at least two hits in two different scintillators in the upper part of the apparatus. The track starting point had to be inside the apparatus. To reject fake semicontained events entering from a detector crack, the extrapolation of the track in the lower part of the detector had to cross and not fire at least three streamer tube planes and one scintillation counter. The measured muon velocity $\beta c$ was evaluated with the same convention of upgoing muons. The range of the IU signal is $-1.3 \leq 1 / \beta \leq-0.7$. After the subtraction of background events, mainly due to wrong time measurements or secondary particles hits, we had 157 upgoing partially contained events.

The zenith distribution of the IU events, compared to the no oscillation prediction, shows a global reduction in the flux of these events, without any appreciable distortion in the shape, Fig. a $21 \%$ systematic scale error. Notice that at these energies the Bartol, the new HKKM and FLUKA fluxes agree also in absolute values.

The identification of ID+UGS events was based on topological criteria. The candidates had a track starting (ending) in the lower apparatus and crossing the bottom detector face. The track had also to be located or oriented in such a way that it could not have entered (exited) undetected through insensitive regions of the apparatus. For this analysis the effective livetime was 5.6 yrs. The event selection required the presence of one reconstructed track crossing the bottom layer of the scintillators and that all hits along the track were confined one meter inside each MACRO supermodule. To reject ambiguous or wrongly tracked events passing the selection, a scan with the Event Display was performed. After 


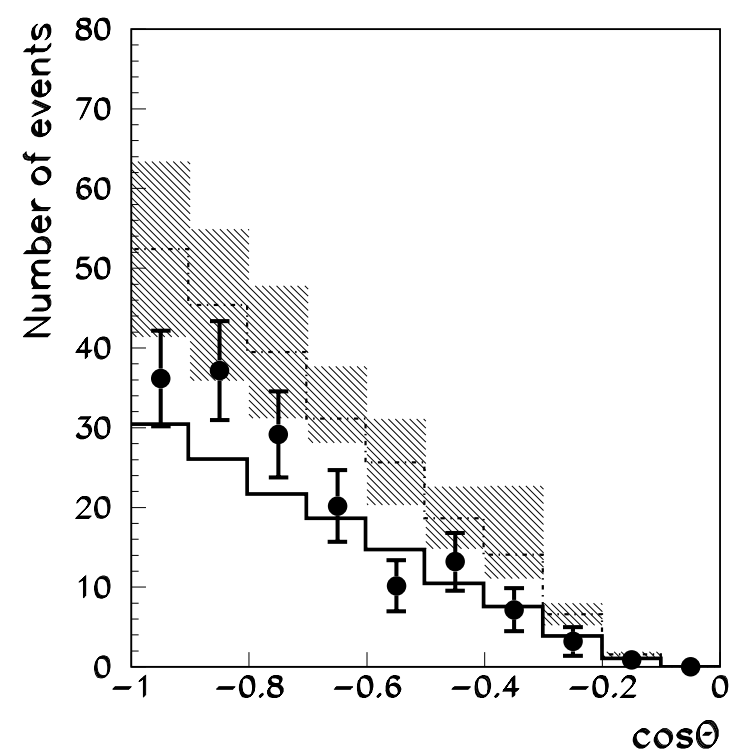

(a)

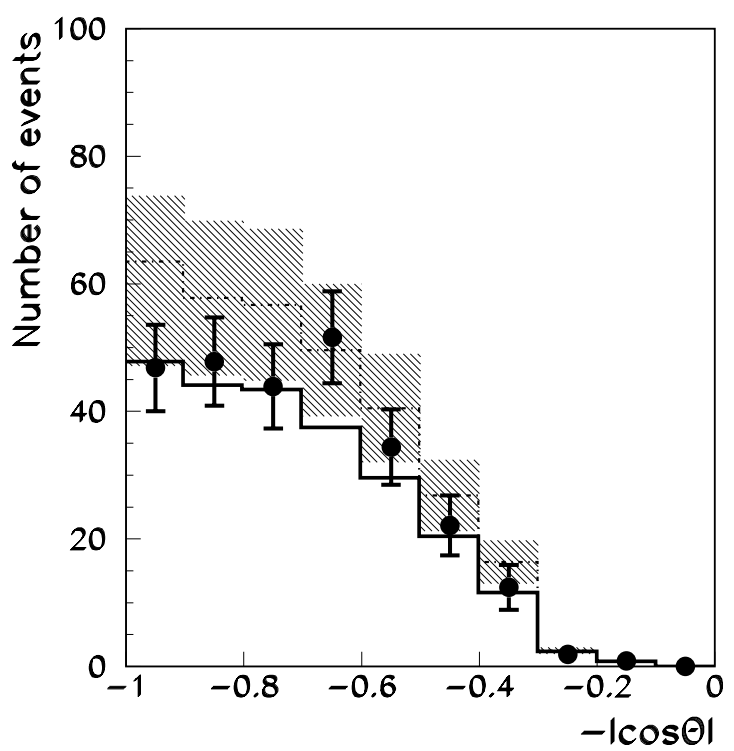

(b)

Figure 4: Measured zenith distributions (a) for the IU events and (b) for the ID+UGS events. The black points are the data, the dashed lines and the shaded regions correspond to MC predictions assuming no oscillations. The full line is the expectation for $\nu_{\mu} \longleftrightarrow \nu_{\tau}$ oscillations with maximal mixing and $\Delta m^{2}=2.3 \cdot 10^{-3} \mathrm{eV}^{2}$.

background subtraction, we had 262 ID+UGS events. The zenith distribution shows, as expected, a uniform deficit of about $25 \%$ of the measured number of events with respect to the no oscillation predictions, Fig.

\section{Determination of the oscillation parameters}

Many oscillation mechanisms have been suggested to explain the atmospheric neutrino data. Here only the two flavor mechanism is examined; after excluding at $99.8 \%$ C.L. the $\nu_{\mu} \longleftrightarrow \nu_{\text {sterile }}$ oscillations (see Sect. $\overline{4} . \overline{1} . \overline{2}$ ), we assume that the contribution of channels

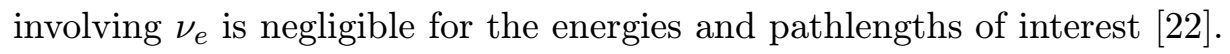

In order to reduce the effects of the uncertainties in the MC simulations (to about 6\%) we used ratios between numbers of events belonging to different categories. To test the no oscillation hypothesis and to evaluate the sensitivity, we considered the following three independent ratios :

1. $R_{1}=N_{\text {vert }} / N_{\text {hor }}$ (see Sect. 4.1.1.2. $)$ in the high energy sample. This is the best ratio of counts in different angular ranges for discriminating between the no oscillation and the two flavour oscillation hypotheses [2iㅣㅁ. The error affecting this ratio is about $6 \%$.

2. $R_{2}=N_{\text {low }} / N_{\text {high }}$ (see Sect. $4 . \overline{1}_{1}^{\prime}$ ) of the low energy and high energy upthroughgoing muons, separated using the energy information from the multiple scattering of muons. The combined error in this ratio is about $17 \%$. This large uncertainty, probably overestimated, arises mainly from the primary CR spectrum. 


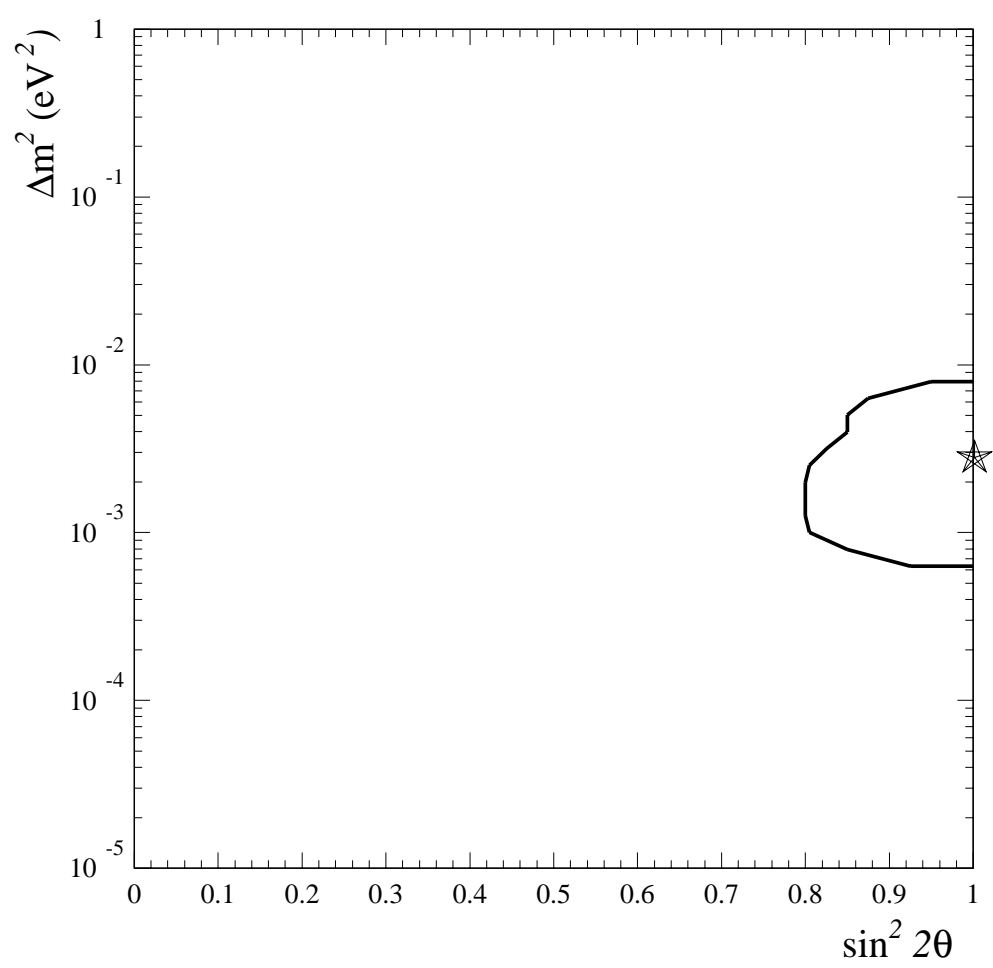

Figure 5: The 90\% C.L. region computed combining the angular distribution of upthroughgoing events, the low energy topologies and the high and low energy subsamples of upthroughgoing events. The star indicates the highest probability point.

3. $R_{3}=N_{I U} / N_{I D+U G S}$ between events in the low energy categories. The combined error on this ratio is about $6 \%$.

\begin{tabular}{ccccccc}
\hline Category & Events & $R_{\text {meas }} \pm \sigma_{\text {stat }}$ & $R_{\tau}$ & $R_{0} \pm \sigma_{\text {sys }}$ & $R_{0} \leq R_{\text {meas }}^{*}$ & $R_{0} \leq R_{\text {meas }}^{* *}$ \\
\hline$R_{1}$ & 547.3 & $1.38 \pm 0.12$ & 1.61 & $2.11 \pm 0.13$ & $4.5 \cdot 10^{-5}$ & $6.4 \cdot 10^{-6}$ \\
$R_{2}$ & 100.5 & $0.85 \pm 0.16$ & 1.00 & $1.50 \pm 0.25$ & $1.9 \cdot 10^{-2}$ & $7.7 \cdot 10^{-3}$ \\
$R_{3}$ & 418.4 & $0.60 \pm 0.06$ & 0.56 & $0.74 \pm 0.06$ & $4.3 \cdot 10^{-2}$ & $3.1 \cdot 10^{-2}$ \\
\hline Combination & & & & & $6 \cdot 10^{-6}$ & $3.4 \cdot 10^{-7}$ \\
\hline
\end{tabular}

Table 2: Ratios between different event categories: $R_{\text {meas }}$ is the measured value; $R_{\tau}$ is the theoretical value expected for $\nu_{\mu} \longleftrightarrow \nu_{\tau}$ oscillations with $\Delta m^{2}=2.3 \cdot 10^{-3} \mathrm{eV}^{2}$ and maximal mixing; $R_{0}$ is the expected value for no oscillations (the errors are only indicative). The last two columns give the one-sided probability compatible with a statistical fluctuation computed according to the $(*)$ and $\left({ }^{*}\right)$ assumptions explained in the text.

The first two ratios were obtained using MC simulations to find the estimators with the best performances in separating the oscillation and no oscillation hypotheses. In principle the ratio $N_{\text {low }} / N_{\text {high }}$ has a better sensitivity to the $\Delta m^{2}$ value; however taking into account the detected number of events and the theoretical uncertainties, the ratio $R_{3}$ is the more statistically significant. 
Table ${ }_{2}^{2}$ shows the measured values of the three ratios and the predictions for no oscillations. In the last two columns the corresponding probability values are given, obtained by allowing the predicted ratios to fluctuate around the mean values and taking into account the non Gaussian distributions of the ratios. The fluctuations were introduced using either the observed number of events $(*)$ or the predicted number without oscillations $(* *)$. Combining the three independent results, we obtain a probability of $6 \cdot 10^{-6}(\sim 5 \sigma)$ that $R_{0} \leq R_{\text {meas }}$.

To take into account the physical boundaries of the oscillation parameters the FeldmanCousins procedure [23i] was used. The result is shown in Fig. $\overline{5}$. We obtained $\chi^{2} / d o f=$ $35.3 / 11$ for no oscillations. The highest probability $\left(\chi^{2} / d o f=10.05 / 11\right)$ is reached at $\Delta m^{2}=2.3 \cdot 10^{-3} \mathrm{eV}^{2}$ and $\sin ^{2} 2 \theta=1$. The first and most important term of $\chi^{2} /$ dof (angular distribution only) is 27.6/9 for no oscillations and 9.5/9 at the point corresponding to the maximum probability.

In the past we used also the total number of events to test the neutrino oscillation

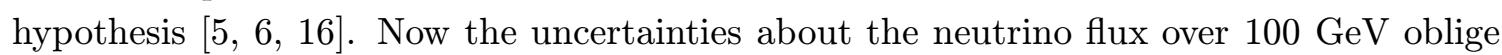
us to give up this test. We do not use the normalization even for low energies in order to be consistent. Anyway we verified that the absolute value of the low energy data gives an additional small contribution in favor of oscillation, confirming the largest probability for the same point and reducing the allowed range on the $\Delta m^{2}$ axis. The same is true for upthroughgoing data if one uses the Bartol flux normalization.

\section{Conclusions}

The MACRO detector took data from 1989 till the end of year 2000. Different analyses have been performed in different energy ranges; all the results rule out the no oscillation hypothesis by $\sim 5 \sigma$ and are compatible with $\nu_{\mu} \longleftrightarrow \nu_{\tau}$ oscillations. The best probability point in the oscillation parameter plane is $\Delta m^{2}=2.3 \cdot 10^{-3} \mathrm{eV}^{2}$ and $\sin ^{2} 2 \theta=1$.

\section{References}

[1] S. Ahlen et al., NNucl. Instrum. Meth. A $\mathbf{3 2 4}(1993) 33$

M. Ambrosio et al., 1Nucl. Instrum. Meth. A 486 (2002) 663 i.

[2] M. Ambrosio et al., 'Eur. Phys. J. C $25-(2002) 511$.

[3] M. Ambrosio et al., 'A strophys.

M. Ambrosio et al., Astropart. Phys. 19 (2003) 1 '."

[4] M. Ambrosio et al., 'P

[5] M. Ambrosio et al., 'P hyss. Lett. B $\mathbf{4} 3 \overline{4}(1998) 451$ '.

[6] M. Ambrosio et al., 'Ph

[7] V. Agrawal et al., 'Phys. Rev. D $\mathbf{5} \mathbf{3}(1996) 13 \overline{1} 14$

[8] M. Gluck et al., 'Z̃.

[9] P. Lipari et al., 'Phys. Rev. Lett. 74 (1995) 384 . 
[10] W. Lohmann et al., "Energy loss of muons in the range 1 - $10000 \mathrm{GeV",} \mathrm{CERN} \mathrm{85-03.}$

[11] R. Brun et al., CERN Publication DD/EE/84-1 (1992).

[12] M. Honda et al., 'Phys. Rev. D 64 (2001) $05301 \overline{1}$.

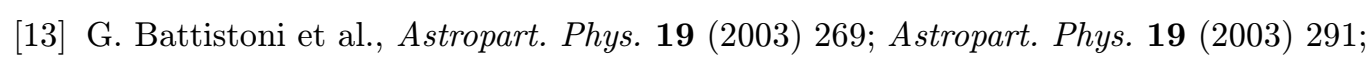

G. Battistoni et al., Proc. of the $28^{t h}$ ICRC, Tsukuba, Japan (2003) 1399.

[14] T.K. Gaisser et al., Proc. of the $27^{\text {th }}$ ICRC, Hamburg, Germany (2001) 1643.

[15] M. Ambrosio et al., Astropart. Phys. 9 $9 \overline{1} 998 \overline{1} 12 \overline{3}$.

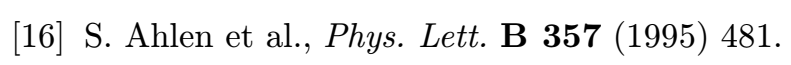

[17] M. Ambrosio et al., Phhys. Lett. B $\mathbf{5 6 6}-2003)$ 35i.

[18] M. Ambrosio et al., №cl. Instrum. Meth. A 321 (1992) 609:

[19] M. Ambrosio et al., №cl. Instrum. Meth. A 492 (2002)376;

[20] P. Lipari and M. Lusignoli Phys. Rev. D 58 (1998) 073005i

[21] M. Ambrosio et al., Phhys. Lett. B $\mathbf{5 1 7}(2001) 599^{\prime}$.

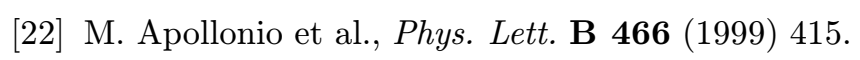

[23] G.J. Feldman and R.D. Cousins, 